\title{
Crouzon's syndrome: Case report and literature review
}

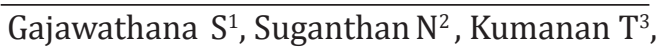 \\ Malaravan $\mathrm{M}^{4}$, Selvaratnam $\mathrm{G}^{5}$
}

\section{Summary}

Crouzon's syndrome is one of the hereditary syndromes of craniofacial dystosis. Patients with these syndromes may be presented to multiple specialties with various presentations such as misshapen head, ocular abnormalities, oro-maxillary defects or even with obstructive sleep apnea or difficulty in breathing due to facial deformities ${ }^{1,2}$. Here, we describe a female who was diagnosed as Crouzon's syndrome in her $5^{\text {th }}$ decade with classical anatomical malformations. She is a 42 year-old unmarried female presented with blurring of vision of left eye for 3 months duration. Her past medical history revealed that she was noticed to have prominent bulging of eyes since birth. When she presented to us, she has flatten occiput with frontal prominence, abnormal contour of head (brachycephaly) and shallow orbit with bilateral pseudoproptosis. She has no hand deformities such as syndactyly or polydactyl as seen with other hereditary craniofacial dystosis syndromes.

\section{Background}

A French neurologist, Octave Crouzon'ss, first described the Crouzon's syndrome in 1912. Crouzon's syndrome is an autosomal dominant disorder with complete penetrance and variable expressivity, caused by mutations in the fibroblast growth factor receptor 2 (FGFR 2) gene and it is characterized by triad of calverial deformities, facial anomalies and exophthalmos. Unlike some other forms of autosomal dominant craniosynostosis, no digital abnormalities are present. Here we present a female who was diagnosed as Crouzon's syndrome in her $5^{\text {th }}$ decade with classical anatomical abnormalities.

\section{Case presentation}

A 42 year-old female presented with insidious onset progressive blurring of vision on left eye for 3 months. The visual loss was painless and was not associated with redness, any significant headache or any other focal neurological signs. Her right eye was normal. She denied any diplopia. She had no any other

\footnotetext{
${ }^{1}$ Registrar in Medicine, University medical unit, Teaching Hospital, Jaffna.

${ }^{2}$ Consultant Physician and senior lecturer, University medical unit, Teaching Hospital Jaffna.

${ }^{3}$ Consultant Physician and senior lecturer, University medical unit, Teaching Hospital Jaffna.

${ }^{4}$ Consultant Eye Surgeon, Teaching Hospital Jaffna.

${ }^{5}$ Consultant Chest Physician and senior lecturer, University medical unit, Teaching Hospital Jaffna.
} 
significant past medical history to note except for some craniofacial deformities since birth. Her family history revealed that no other members in the family were affected by similar abnormalities.

On examination the patient was found to have flatten occiput with frontal prominence, abnormal contour of head (brachycephaly), shallow orbit with bilateral pseudo proptosis, hypertelorism, retruded midface with depressed nasal bridge and prognathic mandible (Fig 1A,B). Oral examination showed normal mouth opening, malocclusion and crowding of maxillary teeth. She did not have any palatal abnormalities such as cleft palate. Ophthalmological examination showed left iris coloboma with cataract. Vision on right eye was normal. She did not have syndactyly or polydactyl. Rest of the neurological examination and other systemic examination were unremarkable. She scored 30 out of 30 in MMSE (mini mental state examination).

On imaging, lateral skull x-ray showed concave profile with retruded and deformed mid facial bone with prominent mandible (Figure 2). CT scan of the brain showed prominent out bulging of the temporal bone causing exophthalmos in addition to the prominent sulci and gyri, lateral ventricles and cisterna magna (Figure 3). Routine hematological and biochemical tests were within normal limits.

A clinical diagnosis of Crouzon's syndrome was made according to the morphological features along with the radiological abnormalities. Genetic studies were not performed due to its unavailability in the public sector.

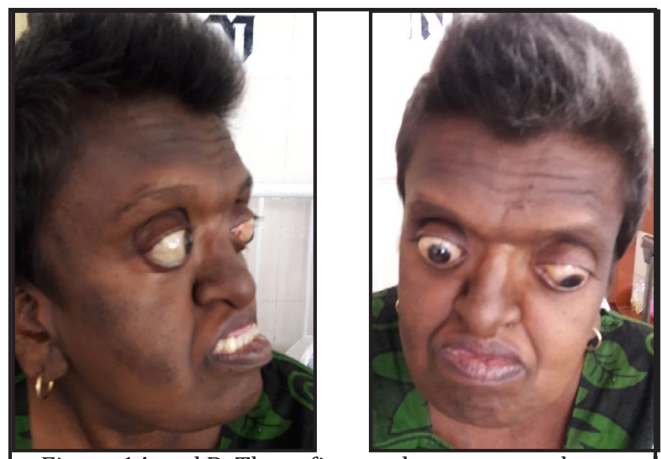

Figure $1 \mathrm{~A}$ and $\mathrm{B}$ : These figures demonstrate abnorma contour of head with bilateral pseudo proptosis hypertelorism, retruded mid face and prognathi mandible.

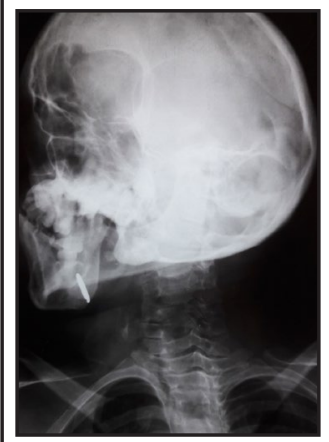

Figure.2

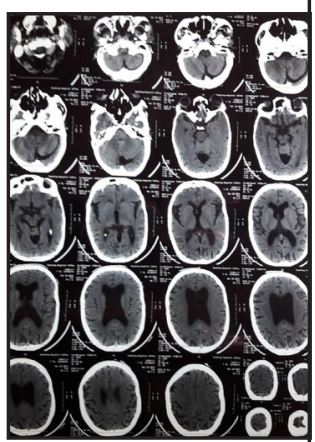

Figure.3
Figure 2 : Lateral skull X-Ray showing the features of concave profile with retruded and deformed mid facial bone and prominent mandible.

Figure 3 : CT brain showing prominent out-bulging temporal bone causing exophthalmos, prominent sulci, prominent bilateral lateral ventricles and prominent cisterna magna.

\section{Discussion}

A French neurologist, Octave Crouzon's, first described the Crouzon's syndrome in 1912. It is one of the varieties of craniosynostosis. It is also called as craniofacial dysostosis ${ }^{3}$. Crouzon's syndrome is an autosomal dominant disorder with complete penetrance and variable expressivity, but about one third of the cases arise spontaneously. The lady described above has no family history of similar condition, therefore this illness in 
our patient is probably due to spontaneous mutations. It occurs due to mutation in the fibroblast growth factor receptor 2 gene on chromosome locus 10q25q26, which results in early fusion of the skull bones during fetal development. Sporadic cases of Crouzon's syndrome has been associated with advanced paternal age ${ }^{4}$. Cranial malformation in Crouzon's syndrome depends on the order and rate or progression of sutural synostosis ${ }^{5}$.

Differential diagnosis of Crouzon's syndrome includes Apert syndrome, Carpenter syndrome, Pfeiffer syndrome, Seatre-Chotzen syndrome, and Jackson Weiss syndrome. Apert syndrome is charecterised by all the manifestations that are seen in Crouzon's syndrome except for the syndactyly of hands and feet. In case of Pfeiffer syndrome broad thumbs, broad big toes and partial and variable soft-tissue syndactyly of hands and feet are the main features in addition to the features of Crouzon's syndrome ${ }^{6}$. Around $30 \%$ of patients also have cervical spine anomalies ${ }^{7}$. But this patient does not have any spinal abnormalities.

Premature synostosis of the coronal, the sagittal, and, occasionally, the lamboid sutures begins in the first year of life and is completed by the second or third year. The order and rate of suture fusion determine the degree of deformity and disability. Once a suture become fused, growth perpendicular to that suture becomes restricted, and the fused bones act as a single bony structure. Compensatory growth occurs at the remaining open sutures to allow continued brain growth. However, multiple sutural synostosis frequently extend to premature fusion of the skull base sutures, causing midfacial hypoplasia, shallow orbits, a foreshortened nasal dorsum, maxillary hypoplasia, and occasional upper airway obstruction $^{2}$.

Early diagnosis is important for the multidisciplinary management of Crouzon's syndrome. Thorough clinical, radiological and genetic analysis is required for early diagnosis. Prenatal diagnostic testing for FGFR gene mutation is an option for couples at risk for having a child with Crouzon's syndrome. Ultrasonic prenatal diagnosis of exophthalmos might give a clue to the developing problem ${ }^{8}$.

In the first year of life, it is preferred to release the synostotic sutures of the skull to allow adequate cranial volume thus allowing for brain growth and expansion. As the child grows, reshaping of skull may need to be repeated to get the best possible results. If necessary, mid-facial advancement and jaw surgery can be done to provide adequate orbital volume and reduce the exophthalmos to correct the occlusion to an appropriate functional position and to provide for a more normal appearance. Depending on the severity of the malformations the prognosis varies.

Our patient was presented to us late with the syndrome and was never treated for it previously.

\section{Take home messages or learning point}

Crouzon's syndrome is one of the rare craniosynostosis syndrome. Early diagnosis and early multidisciplinary management strategies may reduce the deformities, though it cannot be cured completely. It represents 
both an economical and a moral burden to the patients and to the families.

\section{Abbreviations}

FGFR 2 - Fibroblast growth factor receptor 2, CT - Computerized tomography, MMSE - Mini mental state examination

\section{References}

1. Nobuyuki M, Kaneshige S, Yoshihiko F, Tetsuji $\mathrm{U}$, Yoshiaki H, A reflectable case of obstructive sleep apnoea in an infant with crouzon syndrome. Journal of craniofacial surgery. 2004; 15(5); 874-878.

2. Sirotnak J, Brodsky L, Pizzuto M, Airway obstruction in the crouzon syndrome;case report and review of the literature. International journal of Paediatric otorhinolaryngology.1995; 31(2-3); 235-246.
3. Gray TL, Casey T, Selva D, Anderson PJ, David DJ. Ophthalmic sequaley of Crouzon's syndrome. Ophthalmology.2005; 112; 1129-34.

4. Rivka L. Glaser, Wen Jiany, Simeon A. Boyadjiev, Alissa K. Tran, Andrea A. Zachary, Lionel Van Maldergem, David Johnson, Sinead Walsh, Michael Oldridge, Steven A. Wall, Andrew O.M. Wilkie, Ethylin Wany Jabs. Paternal origin of FGFR 2 mutations in sporadic cases of crouzon syndrome and Pfeifer syndrome. ScienceDirect. 2000; 66(3); 768-777.

5. Gorlins text book of syndrome of Head and Neck $4^{\text {th }}$ edition.

6. Kaur H, Waraich HS, Sharma CM. Crouzon's syndrome; a case repor and review of literature. Indian journal of Otorlaryngology Head Neck surgery. 2006; 58; 381-82.

7. Bowling EL, Burstein FD. Crouzon's syndrome. Optometry. 2006; 77; 217-22.

8. Leo MV, Suslak L, Ganesh VL, Adnate A, Apuzzio JJ. Crouzon's syndrome; prenatal ultrasound diagnosis by binocular diameters, Europe PMC, obstetrics and gynaecology; 1991; 78; 906-908. 